Research Article

\title{
Scale Invariant Feature Transformation Algorithm-Based Magnetic Resonance Imaging-Assisted Diagnosis of Patients with Stroke and Rehabilitation Nursing
}

\author{
Guoying Liu \\ Department of Disinfection Supply Center, Xi'an Hospital of Traditional Chinese Medicine, Xi'an 710000, Shaanxi, China \\ Correspondence should be addressed to Guoying Liu; liuguoying@xazzy.cn
}

Received 6 April 2021; Revised 6 May 2021; Accepted 24 May 2021; Published 31 May 2021

Academic Editor: Gustavo Ramirez

Copyright ( 2021 Guoying Liu. This is an open access article distributed under the Creative Commons Attribution License, which permits unrestricted use, distribution, and reproduction in any medium, provided the original work is properly cited.

\begin{abstract}
The aim of this paper was to explore the effects of magnetic resonance imaging (MRI) diagnosis in the treatment and rehabilitation of stroke patients. 80 stroke patients admitted to hospital were selected for the experiment and divided randomly into a control group (group A) and an experimental group (group B), with 40 patients in each group. Patients in group B received MRI examination and rehabilitation training every month under their disease progresses, while patients in group A received routine rehabilitation training instead of MRI examination. The results showed that various indicators of patients, including Fugl-Meyer Assessment (FMA), Berg Balance Scale (BBS), Timed "Up\&Go" test (TUG), 6-minute walking test (6MWT), modified Barthel index (MBI), and stroke-specific quality of life scale (SS-QOL), in group B were better than those in group A. It indicated that personalized rehabilitation training under MRI examination had a better effect on recovery of patients. Besides, the MRI images optimized by scale invariant feature transformation (SIFT) algorithm had better resolution, which were helpful for doctors' diagnosis of disease. MBI scale and SS-QOL showed that the self-care ability and quality of life of patients in group B were better than those of group A. Therefore, the above results indicated that MRI diagnosis could accurately judge disease progress of patients to draw up personalized rehabilitation training plan, which had positive effects on promoting treatment and prognosis of stroke patients.
\end{abstract}

\section{Introduction}

Stroke is an acute cerebrovascular disease caused by different etiology, and its clinical features had acute and persistent onset accompanied by focal neurological function defects including ischemic stroke and hemorrhagic stroke [1-3]. Stroke has the characteristics of high incidence, high mortality, and high disability rate. Studies have shown that sustained rehabilitation therapy in the early stage of stroke plays an important role in reducing disability in stroke patients, and such rehabilitation training generally takes months or even years. Rehabilitation of stroke is a long-term process. Due to limited medical resources, most patients need to be transferred to community hospitals or their houses for rehabilitation after treatment in the acute phase.
The nursing process of each institution is self-formulated, and there is a lack of uniformity in content and form, which makes patients difficult to obtain systematic continuous rehabilitation training [4-6]. Continuous rehabilitation nursing is to extend the rehabilitation training of patients from hospital to community and their houses, and its core content includes the continuity of information, relationship, and management. The continuity of information refers to the consistency of patients' information among different medical institutions [7]. Continuous information can ensure the sharing of patient information among different institutions and promote interinstitutional communication to deliver and timely improve patient's care plan. The continuity of relationship refers to the continuous relationship of treatment and nursing between patients and medical staff, 
and the continuity of management means the continuous management of patients' health status according to the changes of patients' needs [8-10].

Image matching is a critical research object in computer vision and plays a vital role in artificial intelligence, virtual reality, medical diagnosis, and adjuvant therapy. SIFT is an algorithm for detecting and describing target points proposed by David Lowe in 1999, and its superior performance has attracted a large number of scholars for in-depth research. The specific process of SIFT is to first construct the scale space to search for local extremum points for obtaining the main direction of feature points, so as to generate stable descriptors for matching $[11,12]$. SIFT applied in the optimization of medical diagnostic image is beneficial for doctors to make reasonable diagnosis of patients' conditions, so as to provide effective guidance for rehabilitation treatment of stroke patients.

To sum up, SIFT was adopted to patients' MRI images for feature extraction and matching, so as to improve the image quality. Besides, the treatment and rehabilitation effects of patients would be evaluated on this basis.

\section{Materials and Methods}

2.1. Subjects Investigated. A total of 80 stroke patients admitted to hospital were selected as subjects investigated and grouping randomly into group A and B. Patients in group B received MRI scanning once a month to track the disease progresses, and the content of rehabilitation training was adjusted based on their disease progresses. In addition, patients in group A received routine rehabilitation nursing instead of regular MRI examination. The criteria for inclusion were defined to include patients were diagnosed as stroke by brain MRI scanning, were $18-80$ years old, had the first onset of stroke, and signed the informed consents. The criteria for exclusion were defined to include patients suffered from other serious visceral diseases and severe mental illness and had severe visual or language disorders. The experiment had been approved by the Ethics Committee of Hospital. All the patients have signed the informed consents, and the study had been approved by the ethics committee.

\subsection{Summary of Rehabilitation Training for Patients with} Stroke. Patients and their caregivers jointly participated in the rehabilitation, which was launched by the team cooperation mode. Rehabilitation should be started early based on patients' disease progresses, and rehabilitation intervention should be taken for patients with mild to moderate stroke 24 hours after onset. Patients should take a 45 -minute rehabilitation training for a day at least, which was helpful to the recovery of patients' motor function. According to the actual situation, the training intensity moderately increased to improve the rehabilitation effects. The rehabilitation training contained two aspects. One was the means of rehabilitation in the acute phase that included position placement and transfer, joint range of motion training, standing and walking training, breathing training, and shoulder pain prevention. The other was the means of rehabilitation in the recovery phase that included sitting and standing balance training, gravity center transfer, daily life activity training, coordination training, walking training, and active activity ability training. Patients in two groups were assessed timely in terms of motor function, sensory function, and communication ability.

2.3. Outcome Indicators. FMA and BBS were applied to evaluate patients' movement patterns and coordination, TUG and 6MWT were employed to detect patients' functional motor functions, and MBI and SS-QOL were used for evaluating the improvements of daily life activity ability and quality of life of patients after intervention.

2.4. Evaluation Methods. FMA, designed by Swedish scholar Fugl-Meyer, was a quantitative evaluation method of cumulative points, which was only adopted to evaluate sensorimotor function of stroke patients. The content of assessment included five programs (body movement, balance, sensation, range of motion, and pain). Its simplified version was often applied in clinical practice, and the sluggish phase, spasmodic phase, synkinetic phase, partial separation phase, and separation movement phase of one patient were evaluated successively. Each program was divided into three levels, which were scored as 0 points (incomplete), 1 point (partial complete), and 2 points (fully complete), respectively. Besides, the total score of upper limbs was 66 points, total score of lower limbs was 34 points, and total score was 100 points.

BBS, first put forward by Katherine Berg in 1989, included 14 programs such as stand-up, sit-down, upper arm forward, one-round turning, and one leg stand-on. The score range of each program in BBS was $0-4$ points with 56 points of total score. In addition, the higher scores, the better balance. The result of BBS could well predict the occurrence of adverse events such as patients' falling down, and a score below 40 points showed that patients had a risk of falling down. BBS was the most commonly used balance scale in clinical practice, which had high credibility in evaluating the balance function of patients with cerebrovascular diseases.

TUG was an objective method for measuring basic mobility and balance of a patient. The test was quite simple with the subjects getting up from their chairs, walking three meters forward, and returning to their seats. They wore normal shoes, could use a walker, and were allowed to practice once before the test. Results were recorded in seconds, and more than 20 seconds indicated a mobility disorder.

$6 \mathrm{MWT}$ meant that patients could complete the test in a stable state at their own pace, and it was different from the maximum load test and had advantages of high safety and simple operation. Compared to other walking tests, $6 \mathrm{MWT}$ had better toleration and could more reflect activities in daily life. As a functional walking test, it provided feedback on the walking endurance and practical walking levels of stroke patients. 6MWT was one of the most commonly tests for general functional exercise after stroke. 
MBI reflected the degree of patients' self-care ability, and its evaluation content was necessary for daily life. The Chinese version of $\mathrm{MBI}$ had a high reliability among stroke patients. The content of evaluation included ten programs such as eating, dressing, bathing, and walking. The Likert 5level scoring method was adopted with a total score of 100 points. 20 points or less indicated that a patient was completely dependent on others; $21-40$ points showed that a patient had severe functional disorder and were obviously dependent on others; 41-59 points suggested that a patient had a moderate functional disorder and needed others' help to live, and a score was greater than or equaled to 60 points, indicating a patient had basic self-care ability.

SS-QOL was highly targeted and easy to use and had very good reliability, validity, and sensitivity. The SS-QOL included 12 programs such as physical ability, language, emotion, thinking ability, and working ability, which comprehensively evaluated the quality of life of stroke patients. In addition, SS-QOL could be adopted to measure the results of stroke patients and was a widely applied scale to evaluate the quality of life of stroke patients.

2.5. Statistical Methods. SPSS22.0 was for analyzing and processing the data, two-factor repeated measurement analysis of variance was applied to analyze the results of multiple measurements, the two-independent-sample t-test was employed for intergroup comparison, and the least significant difference (LSD) method was used for intragroup pairwise comparison. $P<0.05$ meant there was a statistical meaning, while $P<0.01$ expressed the difference was statistically significant.

\section{Results}

3.1. Magnetic Resonance Imaging Examination. Figure 1 indicates the MRI images of the stroke patients with intracerebral microhemorrhage, as shown in the arrows. After feature extraction, the image contrast was clearer to reflect the lesion better.

3.2. Comparison on Baseline Data of Motor Function and Quality of Life among Patients in the Two Groups before Intervention. Table 1 shows that there was no difference among patients in the two groups in terms of motor function, balance ability, and quality of life before intervention $(P>0.05)$.

\subsection{Comparison on Motor Function Scores of FMA in Patients} from the Two Groups after Intervention. As shown in Table 2 and Figure 2, the motor function scores of patients in the two groups increased gradually with the extension of leaving hospital time, and the differences in the intragroup multifactor comparison were statistically marked $(P<0.001)$. The motor function scores of patients in the two groups were compared 8 weeks and 12 weeks after leaving hospital, respectively, and there were also statistically great differences
$(P<0.001)$, indicating that the FMA motor function score of patients in group B was greater than that of group A.

3.4. Comparison on Balance Ability Scores of BBS in Patients from the Two Groups after Intervention. Figure 3 and Table 3 indicate that the scores of balance ability were improved with the extension of leaving hospital time, and the difference in the intragroup multifactor comparison was statistically remarkable $(P<0.001)$. The balance ability scores of patients in the two groups were compared after 8 weeks and 12 weeks of leaving hospital, respectively, and there were also statistically substantial differences $(P<0.001)$, suggesting that the BBS balance ability score of patients in group $B$ was superior to the score of group A.

\subsection{Comparison on Walking Ability Results of Patients from} the Two Groups after Intervention. As shown in Figure 4 and Table 4, the results of walking ability in patients from both groups decreased with the time of leaving hospital extending, showing that the difference in the intragroup multifactor comparison was statistically obvious $(P<0.001)$. The walking ability of patients in the two groups was compared after 8 weeks and 12 weeks of leaving hospital, respectively, and there were statistically significant meanings $(P<0.001)$, indicating that the recovery of walking ability in patients from group $B$ was better than that of group $A$.

3.6. Comparison on Results of 6MWT in Patients from the Two Groups after Intervention. Figure 5 illustrates that the 6minute walking distance of patients in the two groups was improved with the extension of leaving hospital time, and there was statistically enormous difference in the intragroup multifactor comparison $(P<0.001)$. There were also statistically great meanings in the comparison on walking ability of patients in the two groups 8 weeks and 12 weeks after leaving hospital, respectively $(P<0.05)$, showing that the recovery of walking ability in patients from group $B$ was superior to that of group A.

\subsection{Comparison on Daily Life Activity Ability of Patients in the} Two Groups after Intervention. As shown in Figure 6 and Table 5, the MBI scores of patients in both groups were improved with the time of leaving hospital extending, and the difference in the intragroup multifactor comparison was statistically extreme $(P<0.001)$. The MBI scores of patients in the two groups were compared 8 weeks and 12 weeks after leaving hospital, respectively, and the meanings were statistically substantial $(P<0.001)$, indicating that the recovery of daily life activity ability in patients from group $B$ was better than that of group A.

\subsection{Comparison on Total Score of Quality of Life in Patients in} the Two Groups after Intervention. Figure 7 and Table 6 demonstrate that the SS-QOL scores of patients in the two groups increased with the extension of leaving hospital time, and there was statistically great difference in the 


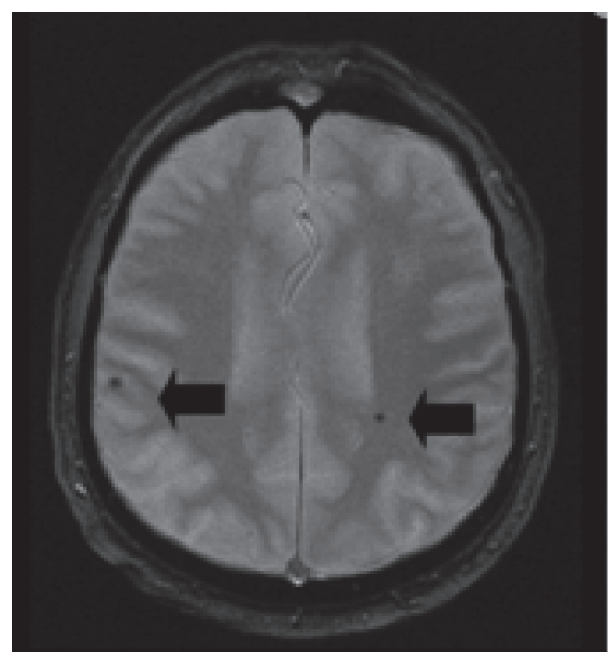

(a)

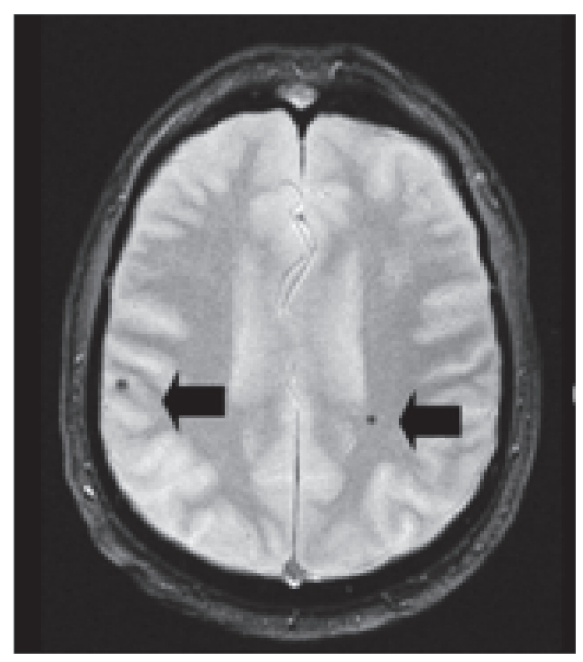

(b)

FIGURE 1: MRI brain images of stroke patients: (a) the original image and (b) the optimized image after SIFT feature extraction.

TABLE 1: Comparison on scores of various indicators in patients from the two groups before intervention.

\begin{tabular}{|c|c|c|c|c|}
\hline Indicators & Group B $(n=40)$ & Group A $(n=40)$ & $\mathrm{t}$ & $P$ \\
\hline FMA & $24.45 \pm 2.12$ & $25.74 \pm 2.19$ & -1.321 & 0.072 \\
\hline BBS & $19.34 \pm 1.98$ & $19.53 \pm 2.01$ & -0.965 & 0.328 \\
\hline TUG & $40.98 \pm 2.09$ & $39.98 \pm 2.12$ & -1.985 & 0.047 \\
\hline $6 \mathrm{MWT}$ & $91.54 \pm 3.98$ & $90.97 \pm 3.54$ & -0.874 & 0.621 \\
\hline MBI & $34.93 \pm 3.62$ & $35.19 \pm 2.89$ & 1.293 & 0.765 \\
\hline SS-QOL & $113.89 \pm 4.23$ & $114.04 \pm 3.57$ & -1.063 & 0.267 \\
\hline
\end{tabular}

TABLE 2: Comparison on FMA motor function scores of patients in the two groups after intervention.

\begin{tabular}{|c|c|c|c|c|c|}
\hline & Baseline & 8 weeks after leaving hospital & 12 weeks after leaving hospital & $\mathrm{F}$ & $P$ \\
\hline Group B & $24.45 \pm 2.12$ & $59.21 \pm 2.01$ & $75.96 \pm 2.26$ & 2704.1 & $<0.001$ \\
\hline Group A & $25.74 \pm 2.19$ & $49.38 \pm 2.09$ & $64.76 \pm 3.65$ & 1398.3 & $<0.001$ \\
\hline $\mathrm{T}$ & -1.321 & 15.384 & 11.324 & - & - \\
\hline$P$ & 0.072 & $<0.001$ & $<0.001$ & - & - \\
\hline
\end{tabular}

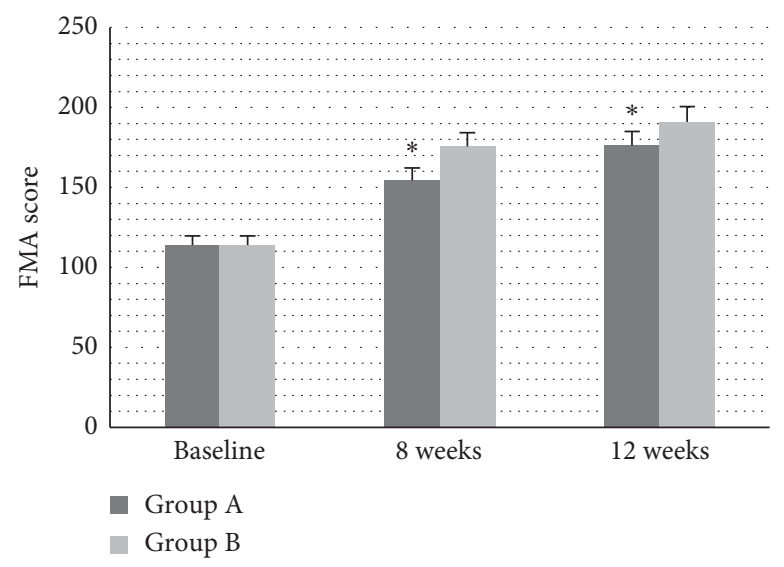

Figure 2: Comparison on FMA motor function scores of patients in the two groups after intervention (*the differences were statistically significant, $P<0.05$, with the same meaning below). 


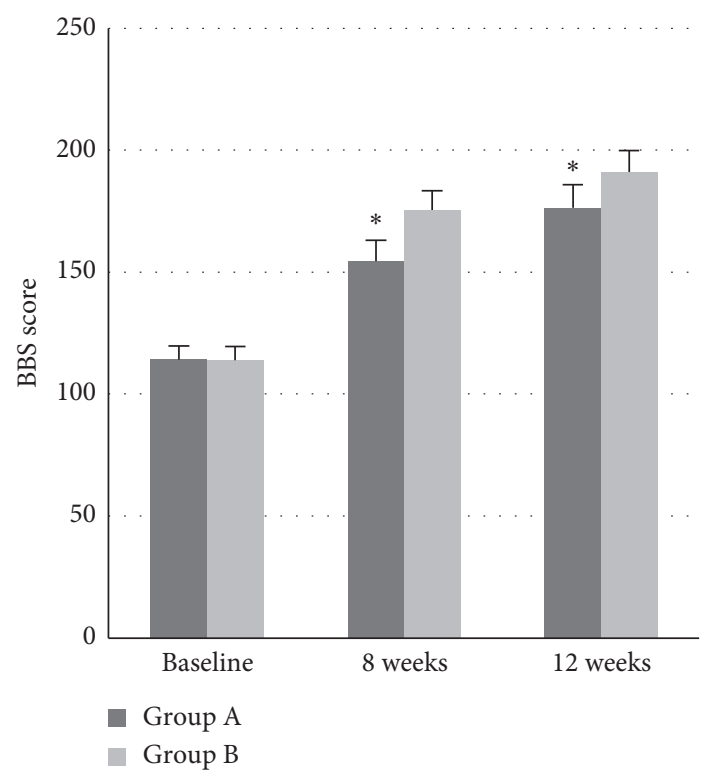

Figure 3: Comparison on FMA motor function scores of patients in the two groups after intervention.

TABLE 3: Comparison on BBS balance ability scores of patients in the two groups after intervention.

\begin{tabular}{|c|c|c|c|c|c|}
\hline & Baseline & 8 weeks after leaving hospital & 12 weeks after leaving hospital & $\mathrm{F}$ & $P$ \\
\hline Group B & $19.34 \pm 1.98$ & $39.21 \pm 2.21$ & $44.96 \pm 2.16$ & 792.1 & $<0.001$ \\
\hline Group A & $19.53 \pm 2.01$ & $34.38 \pm 2.89$ & $37.76 \pm 3.69$ & 398.3 & $<0.001$ \\
\hline $\mathrm{t}$ & -0.574 & 6.384 & 7.324 & - & - \\
\hline$P$ & 0.610 & $<0.001$ & $<0.001$ & - & - \\
\hline
\end{tabular}

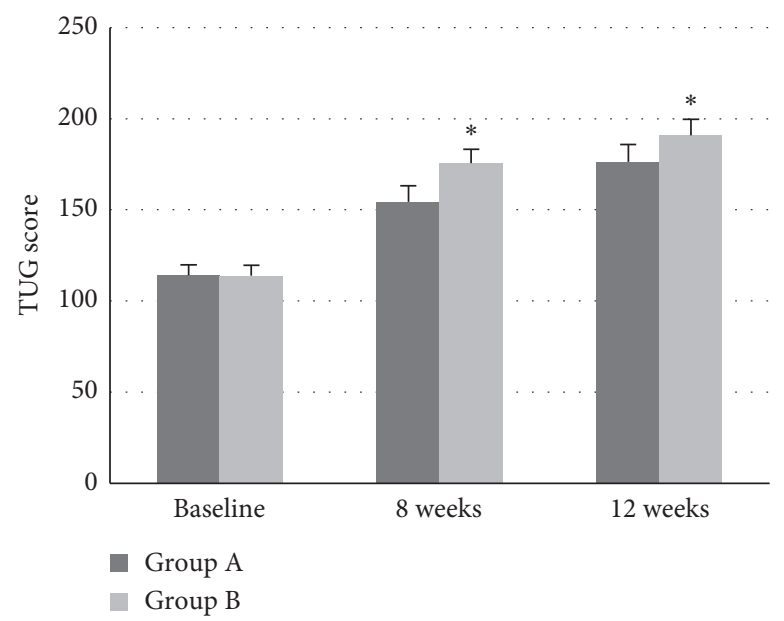

Figure 4: Comparison on TUG scores of patients in the two groups after intervention.

intragroup multifactor comparison $(P<0.001)$. There was also statistically great meaning in the comparison on SSQOL scores of patients in the two groups 8 weeks and 12 weeks after leaving hospital, respectively $(P<0.05)$, so the score of quality of life in patients from group B was higher than the score of group A.

\section{Discussion}

MRI diagnosis was adopted to guide rehabilitation training of stroke patients, so as to obtain good experimental results.
Studies have shown that patients should receive rehabilitation training as soon as possible so that the disease did not progress further. The recovery of stroke patients is closely correlated with the time for rehabilitation, and the first 3 months after the onset of disease are the golden period of physical functional recovery, which can achieve a better recovery effect. The continuity of patients' rehabilitation training can be guaranteed through tracking their conditions. Therefore, it is necessary to grasp the intensity and frequency of rehabilitation activities in a timely manner, so as to effectively promote patients' functional recovery [13-15]. 
TABLE 4: Comparison on results of walking ability in patients in both groups after intervention.

\begin{tabular}{|c|c|c|c|c|c|}
\hline & Baseline & 8 weeks after leaving hospital & 12 weeks after leaving hospital & $\mathrm{F}$ & $P$ \\
\hline Group B & $40.98 \pm 2.09$ & $21.29 \pm 2.21$ & $17.96 \pm 2.16$ & 1092.1 & $<0.001$ \\
\hline Group A & $39.98 \pm 2.12$ & $27.38 \pm 2.89$ & $27.76 \pm 3.69$ & 498.3 & $<0.001$ \\
\hline $\mathrm{t}$ & 1.374 & -6.384 & -13.324 & - & - \\
\hline$P$ & 0.182 & $<0.001$ & $<0.001$ & - & - \\
\hline
\end{tabular}

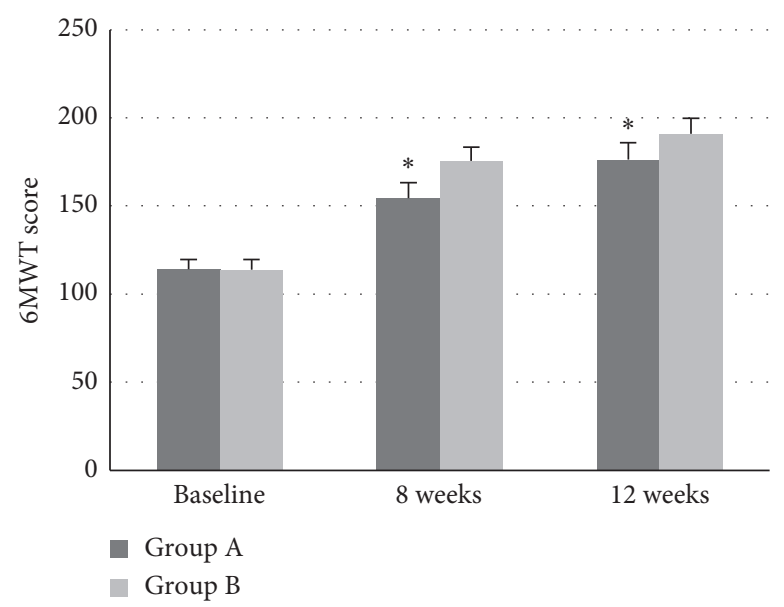

FIgURE 5: Comparison on results of 6MWT in patients from both groups after intervention.

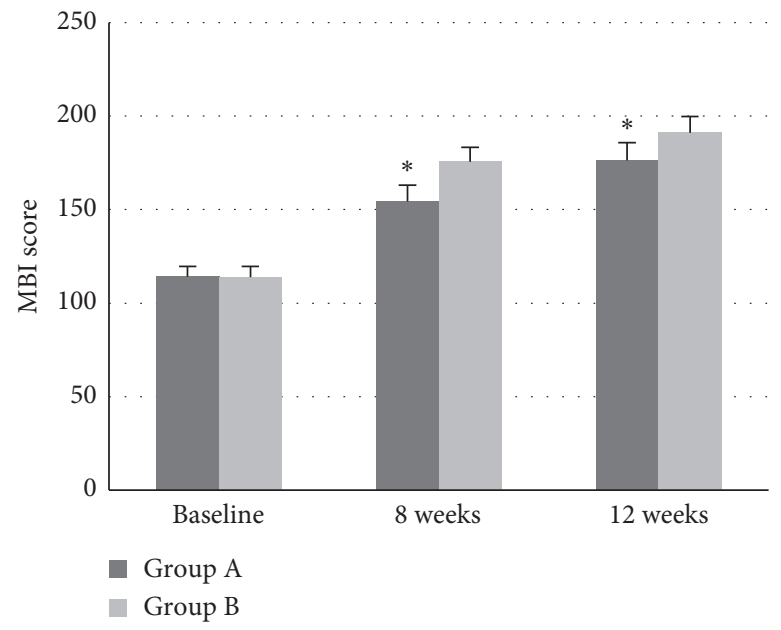

Figure 6: Comparison on MBI scores of patients in the two groups after intervention.

TABle 5: Comparison on MBI scores of patients in both groups after intervention.

\begin{tabular}{|c|c|c|c|c|c|}
\hline & Baseline & 8 weeks after leaving hospital & 12 weeks after leaving hospital & $\mathrm{F}$ & $P$ \\
\hline Group B & $34.93 \pm 3.62$ & $61.55 \pm 2.24$ & $68.96 \pm 2.16$ & 492.1 & $<0.001$ \\
\hline Group A & $35.19 \pm 2.89$ & $57.38 \pm 2.79$ & $61.76 \pm 2.69$ & 308.3 & $<0.001$ \\
\hline $\mathrm{t}$ & -1.074 & 3.109 & 5.324 & - & - \\
\hline$P$ & 0.280 & 0.004 & $<0.001$ & - & - \\
\hline
\end{tabular}

FMA was applied in the study to evaluate the motor function of stroke patients. After rehabilitation training, FMA scores of patients in both groups improved, and the score of patients in group B was better than that of group
A. FMA focuses on the stimulation mode of patients and fails to represent the actual situation of functional activities. BBS scoring assesses stroke patients' balance and coordination. Studies have shown that balance training could 


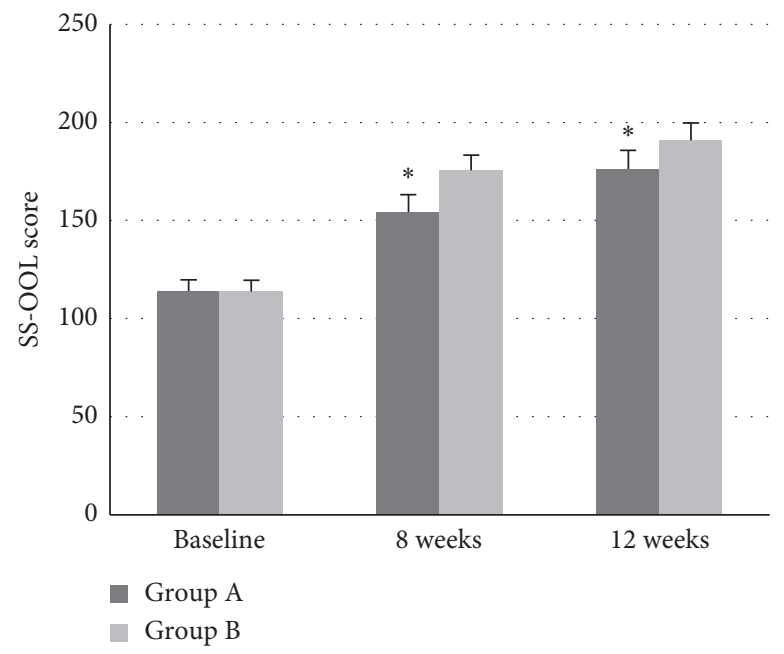

FIGURE 7: Comparison on total score of quality of life in patients from the two groups after intervention.

TABLE 6: Comparison on total score of quality of life in patients from both groups after intervention.

\begin{tabular}{|c|c|c|c|c|c|}
\hline & Baseline & 8 weeks after leaving hospital & 12 weeks after leaving hospital & $\mathrm{F}$ & $P$ \\
\hline Group B & $113.89 \pm 4.23$ & $175.55 \pm 2.24$ & $190.96 \pm 2.86$ & 1492.1 & $<0.001$ \\
\hline Group A & $114.04 \pm 3.57$ & $154.38 \pm 3.79$ & $176.26 \pm 3.69$ & 1308.3 & $<0.001$ \\
\hline $\mathrm{T}$ & -0.574 & 14.109 & 10.324 & - & - \\
\hline$P$ & 0.598 & $<0.001$ & $<0.001$ & - & - \\
\hline
\end{tabular}

effectively improve the physical function of stroke patients and promote the recovery of walking ability. Balance ability can be divided into a static and a dynamic state. Good walking ability and endurance are critical prerequisites for improving the quality of life of stroke patients. TUG and $6 \mathrm{MWT}$ were employed in the study to test the mobility and endurance of patients. After rehabilitation training, the sports ability and endurance of stroke patients in the two groups were enhanced, and the results of patients in group B were superior to those of group A.

\section{Conclusion}

After 3 months of intervention, motor function and coordination of stroke patients were dramatically improved, and their walking and endurance levels were also effectively improved. In contrast to group A, patients in group B could basically take care of themselves in daily life. Moreover, the recovery level of patients in group B was better than that of the group A. The quality of life in patients from group B was superior to that of the group A, as measured by language, activity ability, and family role.

MRI technology develops rapidly these years, which provides objective foundation for the rehabilitation nursing of patients with stroke. However, MRI technology is not yet mature and has not been widely used in clinical practice. In the future, the application of MRI technology should be further explored, to provide guidance for rehabilitation nursing.

In summary, the rehabilitation training plan based on MRI diagnosis could help stroke patients get better treatment and recovery.

\section{Data Availability}

No data were used to support this study.

\section{Conflicts of Interest}

The author declares no conflicts of interest.

\section{References}

[1] C. H. Suh, S. C. Jung, S. J. Cho et al., "MRI for prediction of hemorrhagic transformation in acute ischemic stroke: a systematic review and meta-analysis," Acta Radiologica, vol. 61, no. 7, pp. 964-972, 2020.

[2] Y. Wang, X. Liu, X. Wu, A. J. Degnan, A. Malhotra, and C. Zhu, "Culprit intracranial plaque without substantial stenosis in acute ischemic stroke on vessel wall MRI: a systematic review," Atherosclerosis, vol. 287, pp. 112-121, 2019.

[3] X. Milidonis, I. Marshall, M. R. Macleod, and E. S. Sena, "Magnetic resonance imaging in experimental stroke and comparison with histology," Stroke, vol. 46, no. 3, pp. 843-851, 2015.

[4] A. Gupta, H. Baradaran, A. D. Schweitzer et al., "Carotid plaque MRI and stroke risk," Stroke, vol. 44, no. 11, pp. 3071-3077, 2013.

[5] H. L. Flowers, S. A. Skoretz, D. L. Streiner, F. L. Silver, and R. Martino, "MRI-based neuroanatomical predictors of dysphagia after acute ischemic stroke: a systematic review and meta-analysis," Cerebrovascular Diseases, vol. 32, no. 1, pp. 1-10, 2011.

[6] S. Prabhakaran, I. Ruff, and R. A. Bernstein, "Acute stroke intervention," JAMA, vol. 313, no. 14, pp. 1451-1462, 2015.

[7] A. Rohde, L. Worrall, E. Godecke et al., "Diagnosis of aphasia in stroke populations: a systematic review of language tests," PLoS One, vol. 13, no. 3, p. e0194143, 2018. 
[8] M. Pérez-Piñar, L. Ayerbe, E. González, R. Mathur, Q. FoguetBoreu, and S. Ayis, "Anxiety disorders and risk of stroke: a systematic review and meta-analysis," European Psychiatry, vol. 41, no. 1, pp. 102-108, 2017.

[9] D. Ilunga Tshiswaka, L. E. Sikes, J. Iwelunmor, G. Ogedegbe, and O. Williams, "Transferring stroke knowledge from children to parents: a systematic review and meta-analysis of community stroke educational programs," Journal of Stroke and Cerebrovascular Diseases, vol. 27, no. 11, pp. 3187-3199, 2018.

[10] A. Abdallah, J. L. Chang, C. B. O'Carroll et al., "Stroke in human immunodeficiency virus-infected individuals in SubSaharan Africa (SSA): a systematic review," Journal of Stroke and Cerebrovascular Diseases, vol. 27, no. 7, pp. 1828-1836, 2018.

[11] J. Zhang, G. Chen, and Z. Jia, "An image stitching algorithm based on histogram matching and SIFT algorithm," International Journal of Pattern Recognition and Artificial Intelligence, vol. 31, no. 4, p. 1754006, 2017.

[12] Y. He, G. Deng, Y. Wang et al., "Optimization of SIFT algorithm for fast-image feature extraction in line-scanning ophthalmoscope," Optik, vol. 152, pp. 21-28, 2018.

[13] J. Li, M. Yuan, Y. Liu et al., "Incidence of constipation in stroke patients: a systematic review and meta-analysis," Medicine (Baltimore), vol. 96, no. 25, p. e7225, 2017.

[14] Y. Lian, Y. Zhu, F. Tang et al., "Herpes zoster and the risk of ischemic and hemorrhagic stroke: a systematic review and meta-analysis," PLoS One, vol. 12, no. 2, p. e0171182, 2017.

[15] M. L. A. Nelson, K. A. McKellar, J. Yi et al., "Stroke rehabilitation evidence and comorbidity: a systematic scoping review of randomized controlled trials," Topics in Stroke Rehabilitation, vol. 24, no. 5, pp. 374-380, 2017. 